\title{
Análise sensorial de caldos e canjas elaborados com farinha de carcaças de peixe defumadas: aplicação na merenda escolar ${ }^{+}$
}

\author{
Sensorial analysis of soups and broths made by smoked fish carcasses meal: \\ its utilization to supplement school meals
}

\author{
Leandro Cesar de GODOY ${ }^{1 \star}$, Maria Luiza Rodrigues de Souza FRANCO ${ }^{1}$, Nilson do Prado FRANCO ${ }^{1}$, \\ Adriana Ferreira da SILVA ${ }^{1}$, Michele Ferreira de ASSIS ${ }^{1}$, Nilson Evelázio de SOUZA², \\ Makoto MATSUSHITA², Jesuí Vergílio VISENTAINER²
}

\section{Resumo}

O trabalho avaliou a aceitação de caldos e canjas elaborados com farinhas aromatizadas desenvolvidas a partir de carcaças de peixes defumadas. As carcaças de tilápia do Nilo, carpa e pacu foram lavadas, identificadas, pesadas, imersas em salmoura com ervas aromáticas e defumadas. O produto defumado foi submetido à moagem para obtenção das farinhas, a partir das quais foram elaborados o caldo e a canja. Porções das farinhas, dos caldos e das canjas foram avaliadas por um painel de 40 provadores utilizando-se um método de estímulo simples, sendo avaliados os atributos: aroma, sabor, cor, textura, aparência e aceitação geral. Não houve diferença significativa $(P>0,05)$ na aceitação geral dos produtos. Os caldos elaborados com estas farinhas tiveram uma excelente aceitação, não diferindo significativamente entre si no que se refere aos atributos avaliados. A canja elaborada a partir da farinha de carcaça de pacu apresentou as menores notas quando comparada às demais canjas. Portanto, as farinhas aromatizadas podem ser empregadas no enriquecimento de produtos para o consumo humano. Esses produtos podem ser aplicados na merenda escolar, melhorando a qualidade nutricional das refeições. Além disso, tal uso daria um destino nobre aos resíduos que podem causar sérios impactos se descartados no meio ambiente.

Palavras-chave: resíduo de pescado; sustentabilidade; combate à fome; nutrição humana.

\begin{abstract}
This work evaluated the acceptance of soups and broths prepared with aromatized meals made from smoked fish carcasses. The species of fish used for smoking were Nile Tilapia, carp, and pacu. The carcasses were washed, labeled, weighted, immersed in a solution of brine with aromatic herbs, and smoked. The smoked product was crushed to obtain the meal, which was used to cook the soups and the broths . Portions of meals, broths and soups were sampled by 40 tasters based on a simple stimulus method, which evaluated characteristics such as aroma, flavor, color, texture, aspect, and general acceptance. Considering the three species of fish used to prepare the meals, there were not significant differences $(\mathrm{P}>0,05)$ in the products general acceptance. Soups made with these meals had an excellent acceptance , and so no notable differences in the evaluated attributes were found. The broth made with pacu carcass meal was rated the lowest comparing to the other broths. Therefore, aromatized meals can be used to supplement products destined to human consumption. They can be also used to supplement school meals improving the nutritional quality of children's meal. Such utilization would give a noble destination to residues, which can cause serious impacts if discarded in the environment.
\end{abstract}

Keywords: fish residue; sustainability; hunger combat; human nutrition.

\section{Introdução}

A tilápia do Nilo (Oreochromis niloticus) é o peixe mais cultivado em quase todo Brasil, com mais de 38\% do total produzido; seguida das carpas e dos peixes redondos (tambaqui, pacu e tambacu), ambos representando em torno de $25 \%$ (IBAMA, 2005).

Apesar do grande mercado consumidor, o país apresenta um dos menores índices de consumo per capita de pescado do mundo, tendo apenas a décima posição na América do Sul (FAO, 2005). A média nacional é de 7 a $8 \mathrm{~kg} / \mathrm{hab} / \mathrm{ano}$, quase a metade do recomendado pela Organização Mundial de Saúde $(12 \mathrm{~kg} / \mathrm{hab} / \mathrm{ano})$ e muito inferior à média mundial de $15,8 \mathrm{~kg} / \mathrm{hab} / \mathrm{ano}$. No Brasil, dentre os consumidores de peixes, as crianças são aqueles que apresentam o menor consumo, necessitando de maior trabalho de educação nutricional para estimular o consumo de pescado, melhorando a qualidade da dieta das crianças e surgindo a possibilidade de aplicação na merenda escolar, como sendo uma das estratégias.

+ Este artigo é parte integrante da monografia do autor, a qual recebeu o Prêmio Henri Nestlé de Nutrição e Saúde 2008 na categoria Tecnologia e Bioquímica de Alimentos

${ }^{1}$ Departamento de Zootecnia, Universidade Estadual de Maringá - UEM, Av. Colombo, 5790, CEP 87020-900, Maringá - PR, Brasil, E-mail: godoyzoo@yahoo.com.br'

2 Departamento de Química, Universidade Estadual de Maringá - UEM, Av. Colombo, 5790, CEP 87020-900, Maringá - PR, Brasil

${ }^{*}$ A quem a correspondência deve ser enviada 
Em algumas regiões do Brasil já existem iniciativas, por parte das prefeituras, para inclusão do pescado na merenda escolar. A Secretaria Especial de Aqüicultura e Pesca da Presidência da República (SEAP/PR) e o Fundo Nacional de Desenvolvimento da Educação (FNDE) assinaram em 2007 um acordo de cooperação técnica iniciando, assim, o projeto piloto do Programa de Ampliação do Pescado na Alimentação Escolar, destinado a pescadores artesanais, aqüicultores familiares, merendeiras e conselheiros da alimentação escolar. Este programa tem como objetivo tornar o pescado mais freqüente nas refeições escolares aumentando, desta forma, a sua qualidade nutricional além de proporcionar um aumento na renda da comunidade através da comercialização da produção local de pescado. A criança, além de ser beneficiada com uma alimentação de melhor qualidade, pode desempenhar um papel importante, agindo como agente multiplicador, pois pode incentivar o consumo de pescado também na família.

Os pescados são conhecidos pelo alto valor nutricional, dentre seus constituintes destacam-se o elevado teor protéico, sais minerais (cálcio, fósforo e ferro) e a gordura que é considerada uma das maiores fontes de ácidos graxos da família ômega-3 (n-3). Estes componentes destacam-se pela sua elevada importância fisiológica e nutricional. Estudos realizados por Weaver e Holob (1998) e Simopoulos (1999), comprovaram que o consumo de ácidos graxos poliinsaturados (AGPI) reduz fatores bioquímicos associados à artrite, psoríase e câncer, atuam diretamente no processo de crescimento e desenvolvimento humano e possuem ações antitrombóticas e antinflamatórias.

No Brasil, o aproveitamento dos resíduos de pescado é pequeno, sendo que a maioria destes resíduos é descartada causando um sério problema ambiental. As indústrias de beneficiamento de pescado geram grandes quantidades de resíduos, devido principalmente à falta de reconhecimento deste recurso como matéria-prima e fonte para outros produtos (PESSATTI, 2001). As cabeças, escamas, peles, vísceras e carcaças (esqueleto com carne aderida) são os principais resíduos do processamento de pescado. Dependendo da espécie de peixe processada e do produto final obtido pelo frigorífico, estes resíduos podem representar algo entre 8 a 16\% (no caso do pescado eviscerado) e 60 a $72 \%$ na produção de filés sem pele (KUBITZA, 2006).

A criação de alternativas tecnológicas, com valor agregado que permitam o gerenciamento dos resíduos de pescado, podem trazer como resultado a geração de empregos, o desenvolvimento sustentável e contribuir no combate à fome. O trabalho avaliou a aceitação de caldos e canjas elaborados com farinhas aromatizadas, desenvolvidas a partir de carcaças de tilápia do Nilo, carpa e pacu defumadas.

\section{Material e métodos}

\subsection{Procedimento de defumação e elaboração das farinhas}

Foram utilizadas 100 carcaças - esqueleto após a filetagem, livre de cabeça e nadadeiras - de tilápia do Nilo (Oreochromis niloticus), carpa (Cyprinus carpio) e pacu (Piaractus mesopotamicus) provenientes de pesque-pagues da região de Maringá - PR).
Após serem lavadas e escorridas, as carcaças foram pesadas individualmente, identificadas com placa metálica e imersas em uma solução de salmoura (20\%) com ervas aromáticas (50 g de uma mistura de alecrim, manjericão, salvia, orégano, cebolinha e salsinha desidratadas) por 15 minutos. Em seguida foram penduradas por 1 hora para drenagem e redução da água superficial. Após esta etapa, as carcaças foram colocadas dentro da câmara de defumação, para secagem parcial a uma temperatura de $50^{\circ} \mathrm{C}$, durante 60 minutos. Decorrido esse período, iniciou-se a adição de fumaça. Foi utilizado um defumador artesanal de aço inox, com geração de fumaça externa à câmara de defumação. $\mathrm{O}$ combustível utilizado para produzir a fumaça foi a serragem de eucalipto rosa (Eucalyptus globulus Labill) e, para manutenção da temperatura, utilizou-se gás de cozinha (ASSIS et al., 2009).

A temperatura da defumação iniciou-se $\operatorname{com} 60^{\circ} \mathrm{C}$ e atingiu $80^{\circ} \mathrm{C}$ na última hora de defumação, com acréscimo de $10^{\circ} \mathrm{C}$ a cada hora, totalizando 3 horas de exposição das carcaças à fumaça. $\mathrm{O}$ produto defumado foi submetido à moagem para obtenção das farinhas, embalado em sacos plásticos e estocado sob refrigeração $\left(5^{\circ} \mathrm{C}\right)$ até o momento da realização da análise sensorial, 24 horas do término da defumação.

\subsection{Preparo dos caldos e canjas}

A partir das farinhas foram elaborados dois produtos, o caldo e a canja.

Ambos foram preparados para um volume final de 0,5 L. Os ingredientes utilizados foram: 1 colher (sopa) óleo de soja; 2 colheres (sopa) da farinha aromatizada; 1 colher (café) sal; 0,5 L de água; 1 colher (sopa) polpa de tomate; 1 dente de alho triturado; $1 / 2$ cebola (média) triturada; $1 /{ }_{2}$ colher (sobremesa) de cebolinha $\mathrm{e}^{1 / 2}$ colher (sobremesa) de salsinha. Na elaboração da canja adicionou-se 3 colheres (sopa) arroz cozido aos demais ingredientes.

O preparo seguiu-se da seguinte forma: em uma panela de alumínio, foram refogados o alho e a cebola em óleo quente. Acrescentou-se a polpa de tomate e a água. Após abrir fervura adicionou-se o arroz (para elaboração da canja) e a farinha aromatizada. Por fim, adicionaram-se o sal, a cebolinha e salsinha. O tempo de cozimento foi de 20 minutos.

\subsection{Análise sensorial}

Porções das farinhas, caldos e canjas foram avaliadas por um painel de 40 provadores não treinados, visando atingir o consumidor comum. O projeto de análise sensorial foi aprovado pelo Comitê de Ética em Pesquisa da Universidade Estadual de Maringá, conforme Resolução 196/96.

Os provadores receberam as porções, de maneira aleatória, em pequenos copos plásticos (tipo café) codificados, juntamente com uma ficha para a análise sensorial. A análise sensorial foi baseada no método de estímulo simples (DUTCOSKY, 1996), sendo avaliados os atributos de aroma, sabor, cor, textura, aparência e aceitação geral. Para tanto, utilizou-se uma ficha com escala hedônica de 9 pontos com os extremos 1 (desgostei muitíssimo) e 9 (gostei muitíssimo). Os resultados 
da aceitabilidade dos produtos elaborados foram transformados de acordo com a Equação 1:

$Y=\sqrt{x+0,05}$

cujos valores de $\mathrm{x}$ variam de 1 a 9 e desta forma os resultados de $Y$ estabelecem um intervalo de 3,00 (para a nota máxima $x=9$ ) a 1,02 (para a nota mínima $x=1$ ).

\subsection{Análise estatística}

Foi utilizado um delineamento em blocos casualizados (DBC), onde cada provador foi considerado um bloco. Os resultados foram submetidos à análise de variância (ANOVA, $\alpha=0,05$ ) e as médias foram comparadas pelo teste de Tukey, utilizando-se o software STATISTICA ${ }^{\circledR}$ (STATSOFT Inc., versão 6.0).

\section{Resultados e discussão}

\subsection{Análise sensorial das farinhas aromatizadas}

Não houve diferença significativa $(P>0,05)$ entre as farinhas para as características aroma, sabor e cor (Tabela 1), porém, para aparência e textura a farinha aromatizada obtida a partir da carcaça da carpa recebeu as maiores notas pelos provadores, apesar de não ter diferido significativamente da farinha aromatizada obtida da carcaça de tilápia. Todavia, a farinha obtida a partir da carcaça de pacu não diferiu da farinha de carcaça de tilápia.

Quanto à aceitação geral, as farinhas avaliadas não apresentaram diferença significativa, mostrando que independente da espécie utilizada para obtenção da farinha aromatizada o valor sem transformação corresponde a nota 7 , que significa gostei regularmente do produto. Cabe ressaltar que a nota atribuída pelos provadores é satisfatória, uma vez que as farinhas foram elaboradas com o objetivo de serem adicionadas a outros produtos (caldos, canjas e sopas) e não consumidas de forma pura.

\subsection{Análise sensorial dos caldos e canjas elaborados com as farinhas aromatizadas}

Com relação aos caldos elaborados a partir das diferentes farinhas aromatizadas, não houve diferença significativa $(\mathrm{P}>0,05)$ entre as porções para todos os atributos avaliados (Tabela 2). As notas atribuídas pelos provadores, sem transformação dos dados, correspondem a 8, ou seja, gostei muito. Portanto, pode ser utilizada qualquer uma das três farinhas aromatizadas que o produto a ser elaborado terá boa aceitação.

A canja elaborada a partir das farinhas de tilápia e carpa não diferiram significativamente para todos os atributos avaliados (Tabela 3). Já a canja elaborada a partir da farinha de pacu apresentou as menores notas quando comparada às demais canjas, exceto para o atributo textura, o qual não diferiu significativamente entre as canjas.
Tabela 1. Valores médios transformados das notas atribuídas pelos provadores das farinhas na análise sensorial.

\begin{tabular}{llccccc}
\hline Espécie & Aroma & Sabor & Aparência & Textura & Cor & $\begin{array}{c}\text { Aceitação } \\
\text { geral }\end{array}$ \\
\hline Tilápia & $2,55^{\mathrm{a}}$ & $2,47^{\mathrm{a}}$ & $2,49^{\mathrm{ab}}$ & $2,49^{\mathrm{ab}}$ & $2,52^{\mathrm{a}}$ & $2,55^{\mathrm{a}}$ \\
Carpa & $2,57^{\mathrm{a}}$ & $2,41^{\mathrm{a}}$ & $2,55^{\mathrm{a}}$ & $2,58^{\mathrm{a}}$ & $2,52^{\mathrm{a}}$ & $2,57^{\mathrm{a}}$ \\
Pacu & $2,59^{\mathrm{a}}$ & $2,44^{\mathrm{a}}$ & $2,44^{\mathrm{b}}$ & $2,39^{\mathrm{b}}$ & $2,51^{\mathrm{a}}$ & $2,54^{\mathrm{a}}$ \\
Teste F & $0,33^{\text {ns }}$ & $0,57^{\text {ns }}$ & $5,88^{\star}$ & $5,64^{*}$ & $0,13^{\text {ns }}$ & $0,13^{\text {ns }}$ \\
C.V (\%) & 8,64 & 11,3 & 5,44 & 10,01 & 5,13 & 9,19 \\
\hline
\end{tabular}

ns: não significativo $(\mathrm{P}>0,05){ }^{*}$ significativo $(\mathrm{P}<0,05)$. Médias na mesma coluna com a mesma letra não diferem pelo Teste de Tukey $(\mathrm{P}>0,05)$.

Tabela 2. Valores médios transformados das notas atribuídas pelos provadores na análise sensorial do caldo.

\begin{tabular}{llcllcc}
\hline Espécie & Aroma & Sabor & Aparência & Textura & Cor & $\begin{array}{c}\text { Aceitação } \\
\text { geral }\end{array}$ \\
\hline Tilápia & $2,79^{\mathrm{a}}$ & $2,77^{\mathrm{a}}$ & $2,81^{\mathrm{a}}$ & $2,77^{\mathrm{a}}$ & $2,79^{\mathrm{a}}$ & $2,86^{\mathrm{a}}$ \\
Carpa & $2,81^{\mathrm{a}}$ & $2,86^{\mathrm{a}}$ & $2,81^{\mathrm{a}}$ & $2,78^{\mathrm{a}}$ & $2,79^{\mathrm{a}}$ & $2,90^{\mathrm{a}}$ \\
Pacu & $2,78^{\mathrm{a}}$ & $2,82^{\mathrm{a}}$ & $2,77^{\mathrm{a}}$ & $2,80^{\mathrm{a}}$ & $2,71^{\mathrm{a}}$ & $2,86^{\mathrm{a}}$ \\
Teste F & $0,09^{\text {ns }}$ & $0,56^{\text {ns }}$ & $0,19^{\text {ns }}$ & $0,09^{\text {ns }}$ & $0,66^{\text {ns }}$ & $0,27^{\text {ns }}$ \\
C.V $(\%)$ & 9,13 & 10,56 & 9,44 & 9,25 & 10,83 & 6,41 \\
\hline
\end{tabular}

ns: não significativo $(\mathrm{P}>0,05)$. Médias na mesma coluna com a mesma letra não diferem pelo Teste de Tukey $(\mathrm{P}>0,05)$.

Tabela 3. Valores médios transformados das notas atribuídas pelos provadores na análise sensorial da canja.

\begin{tabular}{lcccccc}
\hline Espécie & Aroma & Sabor & Aparência & Textura & Cor & $\begin{array}{c}\text { Aceitação } \\
\text { geral }\end{array}$ \\
\hline Tilápia & $2,78^{\mathrm{a}}$ & $2,76^{\mathrm{ab}}$ & $2,54^{\mathrm{ab}}$ & $2,56^{\mathrm{a}}$ & $2,67^{\mathrm{a}}$ & $2,68^{\mathrm{ab}}$ \\
Carpa & $2,69^{\mathrm{a}}$ & $2,81^{\mathrm{a}}$ & $2,68^{\mathrm{a}}$ & $2,69^{\mathrm{a}}$ & $2,74^{\mathrm{a}}$ & $2,83^{\mathrm{a}}$ \\
Pacu & $2,45^{\mathrm{b}}$ & $2,51^{\mathrm{b}}$ & $2,24^{\mathrm{b}}$ & $2,51^{\mathrm{a}}$ & $2,22^{\mathrm{b}}$ & $2,44^{\mathrm{b}}$ \\
Teste F & $6,32^{\star}$ & $3,97^{\star}$ & $5,06^{\star}$ & $1,64^{\mathrm{ns}}$ & $15,85^{\star}$ & $6,02^{\star}$ \\
C.V (\%) & 12,47 & 14,15 & 19,33 & 13,29 & 13,38 & 14,28 \\
\hline
\end{tabular}

ns: não significativo $(\mathrm{P}>0,05)^{\star}$ significativo $(\mathrm{P}<0,05)$. Médias na mesma coluna com a mesma letra não diferem pelo Teste de Tukey $(\mathrm{P}>0,05)$.

Analisando a aceitação geral, pode-se verificar que os provadores gostaram muito (nota 8) das canjas elaboradas a partir das farinhas de carpa e tilápia e gostaram regularmente (nota 7) da canja elaborada a partir da farinha de pacu. Stevanato et al. (2007) avaliando a sopa elaborada a partir da farinha de cabeças de tilápia do Nilo obteve excelente aceitação pelos provadores. Já o caldo elaborado a partir da mesma farinha não apresentou boa aceitação, segundo o autor, pelo fato da sua formulação só apresentar a farinha de cabeças, água e sal.

\section{Conclusões}

Os caldos e as canjas elaborados a partir das farinhas aromatizadas foram bem aceitos pelos consumidores. Independente da espécie utilizada, a farinha aromatizada pode ser empregada no enriquecimento de produtos para o consumo humano. Esses produtos podem ser aplicados na merenda escolar, contribuindo na nutrição das crianças. Além disso, tal uso daria um destino nobre aos resíduos que podem causar sérios impactos se descartados no meio ambiente. 


\section{Referências bibliográficas}

ASSIS, M. F.; FRANCO, M. L. R.; STEFANI, M. V.; FRANCO, N.; GODOY, L. C.; OLIVEIRA, A. C.; VISENTAINER, J. V.; SILVA, A. F.; HOCH, A. L. V. Efeito do Alecrim na Defumação da Carne de Rã (Rana catesbeiana): Características Sensoriais, Composição e Rendimento. Ciência e Tecnologia de Alimentos, v. 29, n. 3, p. 553-556, 2009.

DUTCOSKY, S. D. Análise sensorial de alimentos. Curitiba: Champagnat, 1996. 123 p.

FOOD AND AGRICULTURE ORGANIZATION - FAO. Yearbook of fishery statistics: summary tables. Roma, 2005. Disponível em: <http://www.fao.org >. Acesso em: abr. 2006.

INSTITUTO BRASILEIRO DO MEIO AMBIENTE E DOS RECURSOS NATURAIS RENOVÁVEIS - IBAMA. Brasília, 2005. Disponível em: <http://www.ibama.gov.br>. Acesso em: abr. 2006.
KUBITZA, F. Aproveitamento dos subprodutos do processamento de pescados. Panorama da Aqüicultura, v. 16, n. 94, p. 23-29, 2006.

PESSATTI, M. L. Aproveitamento dos subprodutos do pescado: meta 11. Santa Catarina: Universidade do Vale do Itajaí, 2001. (Relatório final de ações prioritárias ao desenvolvimento da pesca e aqüicultura no sul do Brasil, convênio Ministério da Agricultura, Pecuária e Abastecimento - MAPA)

SIMOPOUlOS, A. P.; LEAF, A.; SALEM, N. Essentiality and recommended dietary intakes for omega- 6 and omega- 3 fatty acids. Annals of Nutrition and Metabolism, v. 43, p. 127-30, 1999.

STEVANATO, S. B. et al. Avaliação química e sensorial da farinha de resíduo de tilápia na forma de sopa. Ciência e Tecnologia de Alimentos, v. 27, n. 3, p. 567-571, 2007.

WEAVER, B. J.; HOLOB, B. J. Health effects and metabolism of dietary eicosapentaenoic acid. Food Nutrition Science, v. 12, p. 111-150, 1998. 